\title{
Diagnostic and Prognostic Values of Blood Test Parameters in Hand, Foot, and Mouth Disease: A Meta-analysis
}

\author{
Boqiu Zhang ${ }^{1}$ Youcheng Huang ${ }^{1}$ Rongrong Yang ${ }^{1}$ Liu Yang ${ }^{2}$ \\ 1 Pediatrics Department, Fujian Provincial Maternity and Children's \\ Hospital, Affiliated Hospital of Fujian Medical University, Fuzhou, China \\ 2 Unimed Scientific Inc., Wuxi, China \\ J Pediatr Infect Dis 2020;15:176-183. \\ Address for correspondence Boqiu Zhang, PhD, Pediatrics
Department, Fujian Provincial Maternity and Children’s Hospital,
Affiliated Hospital of Fujian Medical University, Fuzhou, China
(e-mail: 13809550620@163.com).
}

\begin{abstract}
Keywords

- HFMD

- meta-analysis

- NT-proBNP

- IL-8

- blood glucose

Objective The present meta-analysis was conducted to identify the diagnostic and prognostic significance of routine blood test parameters in patients with hand, foot, and mouth disease (HFMD), in particular for white blood cell (WBC) count, platelet level, C-reactive protein (CRP), interleukin-8 (IL-8) level, fasting blood glucose level, plasma N-terminal pro-B-type natriuretic peptide (NT-proBNP) levels, etc.

Methods PubMed, Web of Science, Embase, Cochrane Library were searched to identify relevant articles. The potential risk factors were analyzed when patients were divided into groups using different standards. The meta-analyses were performed using the RevMan software. After screening, data from the 26 papers were used for meta-analysis.

Results Some factors with potential diagnostic and prognostic value, and others with no value, were identified. Specifically, WBC counts had an association with later survival; CRP and platelet levels had limited indicative roles; higher plasma glucose levels were associated with more serious complications and poorer survival; fatal progression was correlated with increased IL-8 and NT-proBNP levels. There was great heterogenicity between studies.

Conclusion Blood factors, especially WBC counts, plasma glucose level, IL-8, and NTproBNP levels are associated with the severity and outcome of HFMD.
\end{abstract}

\section{Introduction}

Hand, foot, and mouth disease (HFMD) is a childhood disease mainly caused by enterovirus 71 (EV71) and coxsackievirus A16 (CA16). ${ }^{1,2}$ Outbreaks of HFMD occur in Asia, and the condition largely affects children under 5 years. ${ }^{3}$ The common symptoms include fever $\left(>38^{\circ} \mathrm{C}\right)$, rashes on the volar regions of the hands and feet, and difficulty eating and drinking. In some cases the clinical manifestations are more serious, including herpangina, brain stem encephalitis, and cardiopulmonary systems (e.g., pulmonary edema). These cases can progress dramatically, leading to serious

received

September 4, 2019

accepted after revision

February 8, 2020

published online

March 4, 2020

sequelae or even death. Therefore, identifying factors with diagnostic and prognostic values is worthwhile.

At an early period, patients do not usually undergo testing specifically for EV71 or CA16; routine blood tests are conducted more frequently. Some of these parameters may help with the diagnosis, and assessing the prognosis of HFMD. Theoretically, parameters in the routine blood tests (e.g., white blood cells [WBC]), coagulation tests (e.g., platelet), inflammatory cytokine levels, and other plasma biochemical indexes, may collectively indicate the onset and progression of HFMD. This meta-analysis aimed to identify factors

Copyright (c) 2020 by Georg Thieme Verlag KG, Stuttgart · New York
DOI https://doi.org/ 10.1055/s-0040-1708053. ISSN $1305-7707$. 
(including WBC count, platelet, C-reactive protein [CRP], interleukin-8 [IL-8] level, fasting blood glucose level, etc.) with diagnostic and prognostic significance in HFMD patients. The potential risk factors were analyzed when patients were divided into groups using different standards.

\section{Materials and Methods}

\section{Literature Search}

This review was conducted in accordance with the Preferred Reporting Items for Systematic Reviews and Meta-Analyses (PRISMA) guidelines. We systematically searched data from the all full-published English language papers, excluding meeting or conference abstracts. A thorough literature search was undertaken using the following databases: PubMed, Embase, Cochrane Library, and Web of Science databases. Literatures were searched using the following keywords: "hand, foot, and mouth disease"|"hand-footand-mouth disease"|“HFMD," combined with "patients"| "patient" or "blood" (such as HFMD and patients [abstract], HFMD and patient [abstract], or hand-foot-and-mouth disease and blood [abstract], etc.), with a time range from 1988 to 2018. The union was acquired by combination of the results of: (1) HFMD/hand-foot-and-mouth disease/hand, foot, and mouth disease with patients/patient, and (2) HFMD/hand-foot-and-mouth disease/hand, foot, and mouth disease with blood. The literature retrieval was performed by two independent authors, and quality of the included studies was specifically assessed using the QUADAS tool.

The process for screening is shown in - Fig. 1.

The inclusion criteria were as follows: (1) the studies surveyed factors related to blood tests in HFMD patients; (2) for all clinical studies, patients had to be divided into different groups, e.g., according to severity; (3) studies gave clear diagnostic standards for HFMD.
The exclusive criteria were as follows: (1) duplicate publications; (2) studies not related to HFMD; (3) reports in the forms of conference abstracts, case reports, comments, reviews, or meta-analysis; (4) basic research, rather than clinical studies; (5) studies that did not include detailed data about blood parameters, clear groups for comparison, or otherwise lacked detailed results. Patients in different literatures were grouped according to clinical characteristics: control or HFMD, mild or severe (those with any evidence of central nervous system complications, including aseptic meningitis, encephalitis, and poliomyelitis-like paralysis, or pulmonary edema, were classified into the severe group, or else the mild group), and survived or dead.

The following indexes were observed in different groups, if documented: WBC counts $\left(10^{9} / \mathrm{L}\right)$, platelet $\left(10^{9} / \mathrm{L}\right), \mathrm{CRP}(\mathrm{mg} / \mathrm{L})$, the inflammatory markers IL- $8(\mathrm{pg} / \mathrm{mL})$, fasting blood glucose level (mM), plasma $N$-terminal pro-B-type natriuretic peptide (NT-proBNP) levels, etc. If the data were present as continuous values, at least including mean, SD, and case number, the results were analyzed in the forest plots, or else in the single reference review. The detailed indexes were presented in results.

Data were analyzed with RevMan software (version 5.3; Cochrane Collaboration, Oxford, UK). Mean differences (MDs) with 95\% confidence intervals (CIs) were calculated for the continuous measures. Forest plots were presented after analysis, in which lines represented different estimates and CIs, and boxes represented the weight given to each study. We also quantified the effect of heterogeneity using $I^{2}$. The fixed-effect model was used when no significant heterogeneity existed; otherwise, a random-effect model was used. The fixed-effect model was also calculated for the sensitivity analyses. Substantial heterogeneity across the studies was detected when $I^{2}$ was $>50 \%$ or the $p$-value for heterogeneity was $<0.10$. All the $p$-values were two-sided. The funnel plot

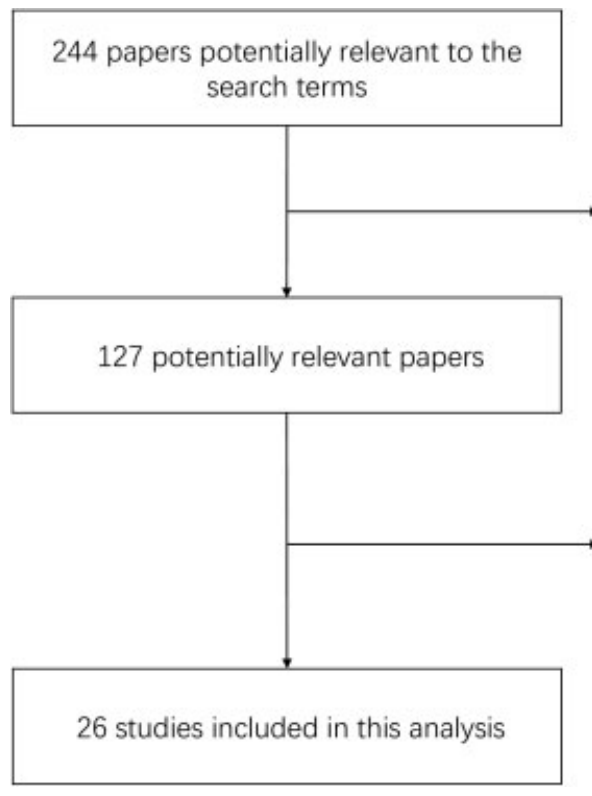

Removing

Replicated studies (112)

Irrelevant studies (5)
Removing

Reviews, Conference abstracts, case reports,

or meta-analysis (4)

Animal, cell or biochemical studies (17)

Insufficient data (80)

Fig. 1 Flow of the study identification, inclusion, and exclusion. 
was used to assess the publication bias. A p-value less than 0.05 was considered to be statistically significant, while the $p$-value less than 0.1 was considered to be significant statistically in heterogeneity analysis.

\section{Results}

\section{Search Results}

A total of 244 papers potentially relevant to the search terms were identified by the initial search (PubMed: 132, Embase: 83 , Cochrane: 29, and Web of Science: 0). Among these 112 duplicate studies were removed, and 5 irrelevant references were excluded. Next, reviews, case reports, or meta-analysis papers (15 papers) were removed. Then, the basic studies were further removed, including animal, cell, or biochemical studies (17 papers), Finally, clinical studies giving insufficient detail of the required indexes were removed ( 80 papers). This left data from 26 papers that were used for the meta-analysis. ${ }^{40}$ All these studies had provided the detailed statistical results of blood test parameters and definitions of different groups. In the QUADAS tools, all studies have provided clear criteria for grouping, but in other items the results of assessment were all "unclear."

\section{Parameters in Routine Blood Tests}

First, the association between the three parameters (WBC counts, CRP, and platelet levels) and severities were analyzed. For WBC, greater severities were related to higher WBC levels. However, references in HFMD-versus-controlcomparison $\left(\mathrm{Tau}^{2}=2.45 ; \mathrm{Chi}^{2}=30.69, \mathrm{df}=5, p<0.0001\right.$; $\left.I^{2}=84 \%\right)$ and severe-versus-mild-comparison ( $\mathrm{Tau}^{2}$ $\left.=8.12 ; \mathrm{Chi}^{2}=122.17, \mathrm{df}=7, \mathrm{p}<0.00001 ; I^{2}=94 \%\right)$ groups

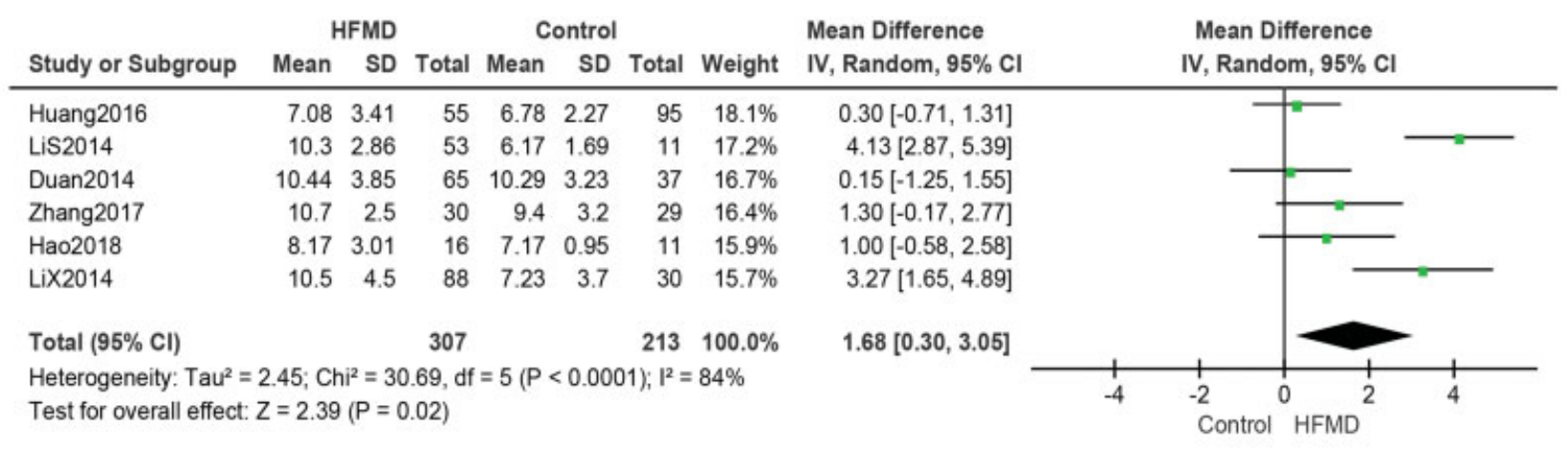

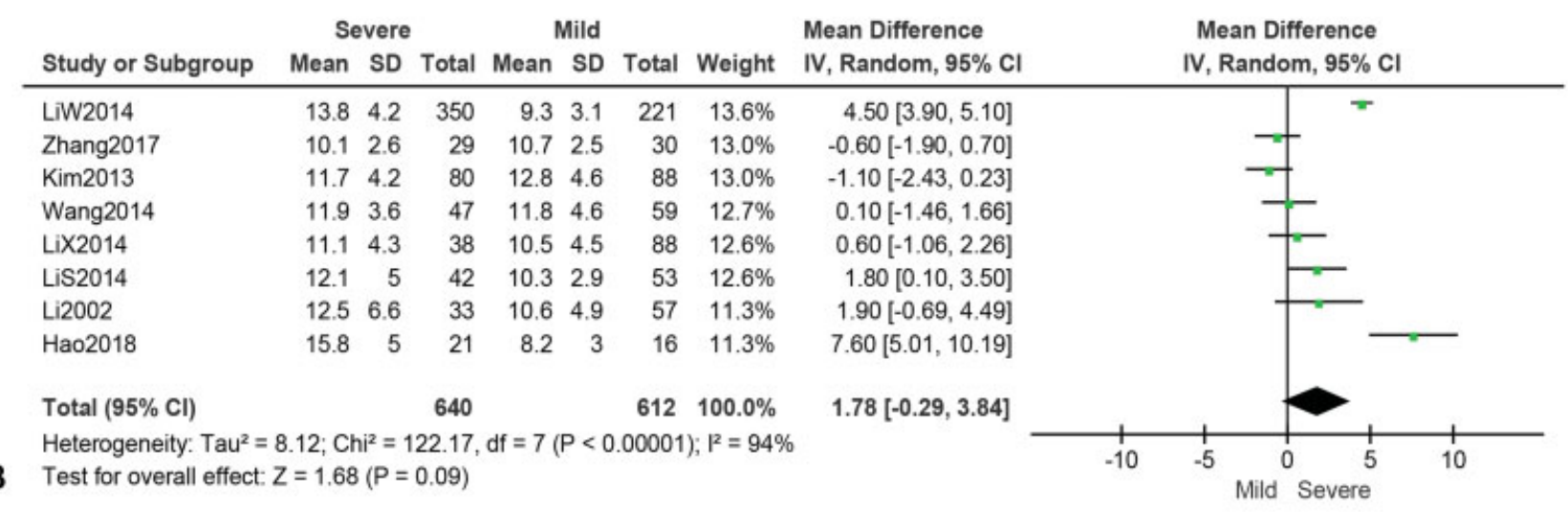

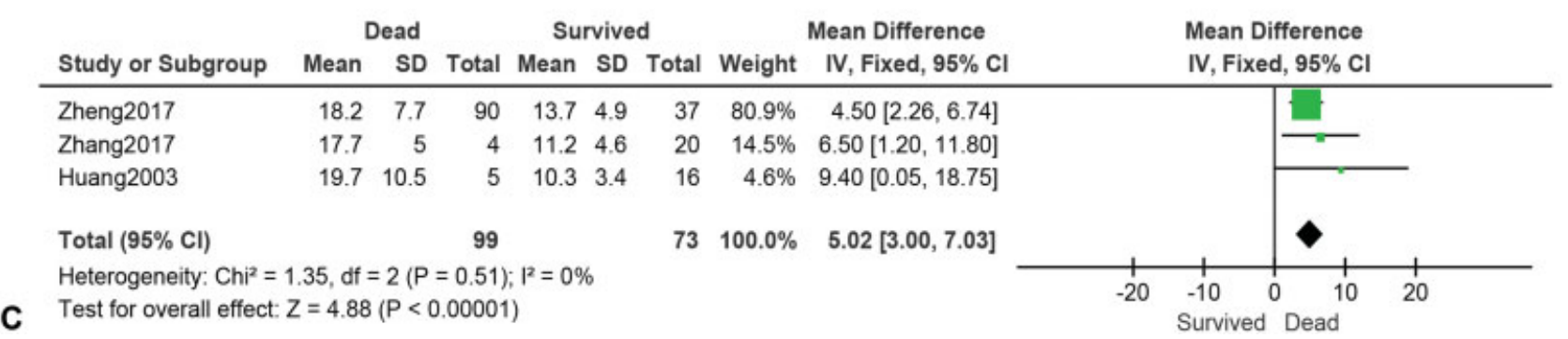

Fig. 2 Cumulative meta-analysis of the white blood cell (WBC) between different groups (presented as forest plots). (A) Difference in WBC counts between hand, foot, and mouth disease (HFMD) and control groups. (B) Difference in WBC counts between the severe and mild groups. (C) Difference in WBC counts between fatal and nonfatal groups. 


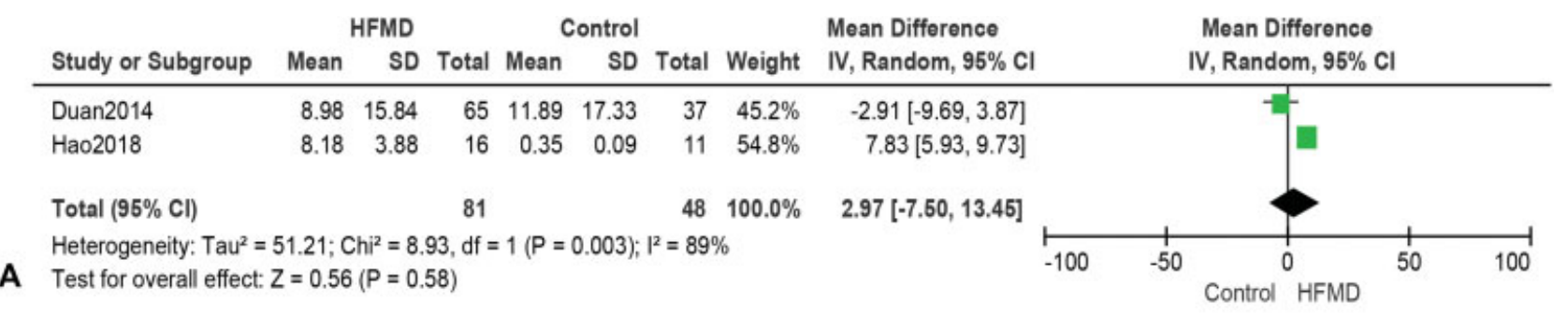

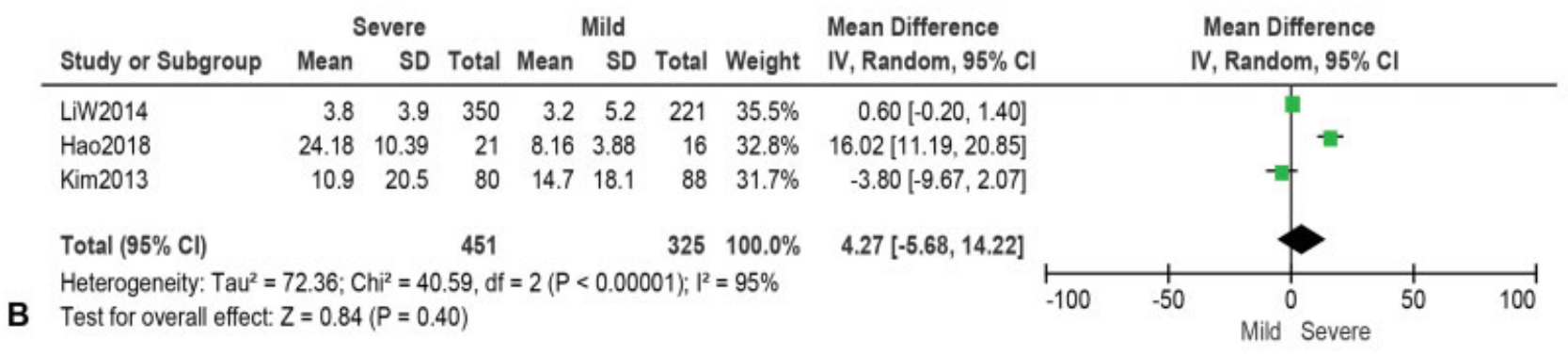

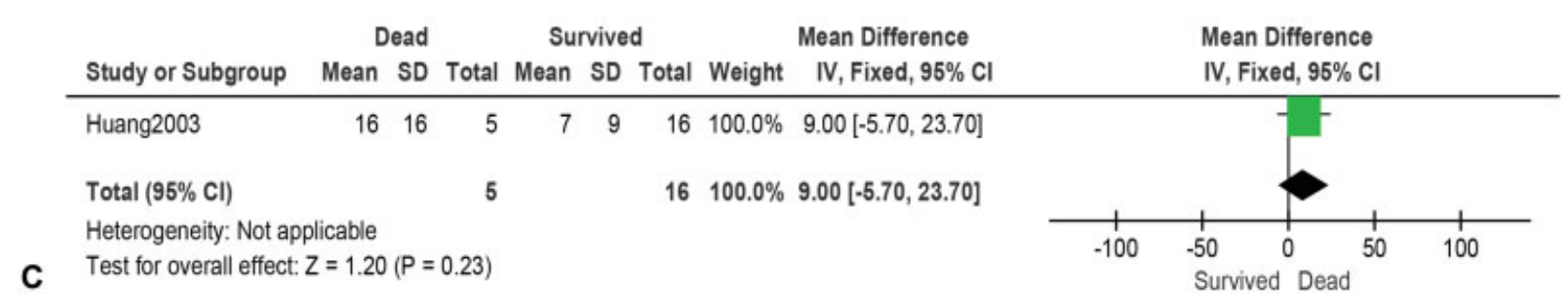

Fig. 3 Cumulative meta-analysis of C-reactive protein (CRP) levels between different groups (presented as forest plots). (A) Difference in CRP expression between HFMD and Control groups. (B) Difference in CRP expression between the severe and mild groups. (C) Difference in CRP expression between fatal and nonfatal groups. HFMD, hand, foot and mouth disease.

showed a significant heterogeneity. Regarding HFMD versus control, WBC counts showed an overall effect between groups $(Z=2.39, p=0.02)$, while there were no effects between severe and mild groups $(Z=1.68$, $p=0.09$; - Fig. $2 \mathrm{~A}$ and $\mathbf{B}$ ). When comparing fatal and nonfatal groups, no heterogeneity was found among studies $\left(\mathrm{Chi}^{2}=1.35, \mathrm{df}=2, p=0.51 ; I^{2}=0 \%\right)$; fatal cases had higher WBC levels than nonfatal ones $(Z=4.88$, $p<0.00001$; -Fig. 2C). CRP levels showed conflicting results among studies in HFMD-versus-control, severeversus-mild, and fatal-versus-nonfatal comparisons (-Fig. 3A-C). In comparison between HFMD and control cases, two studies showed contrary trends $\left(\right.$ Tau $^{2}=51.21$; $\left.\mathrm{Chi}^{2}=8.93, \mathrm{df}=1, p=0.003 ; I^{2}=89 \%\right)$, there is no significant overall effect $(Z=0.56, p=0.58)$; similarly, three studies about CRP differences in severe-versus-mild comparison had distinct results $\left(\mathrm{Tau}^{2}=72.36 ; \mathrm{Chi}^{2}=40.59, \mathrm{df}=2\right.$, $\left.p<0.00001 ; I^{2}=95 \% ; Z=0.84, p=0.40\right)$; also, a single study regarding CRP differences between fatal and nonfatal cases found no association $(Z=1.20, p=0.23)$. The platelet difference between severe and mild was documented in only one study, and no significant difference was found ( - Fig. 4A); the platelet levels between fatal and nonfatal cases showed opposing results in two studies $\left(\mathrm{Tau}^{2}=11075.36\right.$; $\mathrm{Chi}^{2}=8.56$, $\left.\mathrm{df}=1, p=0.003 ; I^{2}=88 \%\right)$, as well with no overall statistical significance $(Z=0.20, p=0.84$; - Fig. 4B). There was significant heterogeneity between studies regarding CRP and platelet levels. However, WBC counts significantly varied between fatal and nonfatal cases.

\section{Plasma Glucose}

The differences in plasma glucose are presented in - Fig. 4. One report showed the HFMD group had slightly higher glucose level than normal but without statistical significance $(Z=1.64$, $p=0.10 ;-$ Fig. 5A). Two consistent results focusing on severeversus-mild comparison showed a significant effect between groups with low heterogeneity $\left(\mathrm{Chi}^{2}=0.58, \mathrm{df}=1, p=0.45\right.$; $I^{2}=0 \% ; Z=2.97, p=0.003 ;$ - Fig. 5B). In addition, four studies regarding the glucose differences between fatal and nonfatal groups had highly identical results $\left(\mathrm{Chi}^{2}=1.48, \mathrm{df}=3\right.$, $\left.p=0.69 ; I^{2}=0 \%\right)$, the fatal cases had elevated plasma glucose levels $(Z=8.44, p<0.00001$; - Fig. $5 C)$.

\section{Interleukin-8}

Among different inflammatory cytokines, we selected only IL-8 as no sufficient data was available for other cytokines. Again, IL-8 expression between HFMD and control groups was controversially reported $\left(\mathrm{Tau}^{2}=582.38 ; \mathrm{Chi}^{2}=3.88\right.$, df 


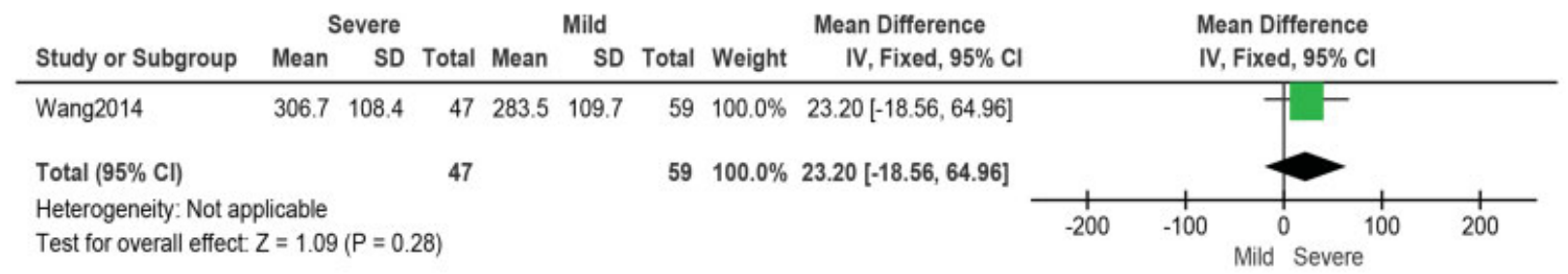

\begin{tabular}{|c|c|c|c|c|c|c|c|c|c|}
\hline \multirow[b]{2}{*}{ Study or Subgroup } & \multicolumn{3}{|c|}{ Dead } & \multicolumn{2}{|c|}{ Survived } & \multirow[b]{2}{*}{ Total } & \multirow[b]{2}{*}{ Weight } & Mean Difference & \multirow{2}{*}{$\begin{array}{c}\text { Mean Difference } \\
\text { IV, Random, } 95 \% \mathrm{Cl}\end{array}$} \\
\hline & Mean & SD & Total & Mean & SD & & & IV, Random, $95 \% \mathrm{Cl}$ & \\
\hline Huang2003 & 423.6 & 94.724 & 5 & 324.625 & 69.483 & 16 & $47.5 \%$ & $98.98[9.24,188.71]$ & \\
\hline Zheng2017 & 401.1 & 113.4 & 90 & 460.5 & 160.1 & 37 & $52.5 \%$ & $-59.40[-116.06,-2.74]$ & \\
\hline Total $(95 \% \mathrm{Cl})$ & & & 95 & & & 53 & $100.0 \%$ & $15.81[-139.20,170.82]$ & \\
\hline \multicolumn{8}{|c|}{ Heterogeneity: Tau $^{2}=11075.36 ; \mathrm{Chi}^{2}=8.56, \mathrm{df}=1(\mathrm{P}=0.003) ; \mathrm{I}^{2}=88 \%$} & Test for overall effect: $Z=0.20(P=0.84)$ & 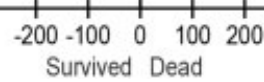 \\
\hline
\end{tabular}

Fig. 4 Cumulative meta-analysis of platelet levels between different groups (presented as forest plots). (A) Difference in platelet levels between the severe and mild groups. (B) Difference in platelet levels between fatal and nonfatal groups.

$=1, p=0.05 ; I^{2}=74 \%$ ), and no differences were found between groups $(Z=0.46, p=0.65$; - Fig. 6A). However, plasma IL-8 expression was significantly higher in the severe group than the mild group, as two studies consistently reported $\left(\mathrm{Chi}^{2}=1.47, \quad \mathrm{df}=1, \quad p=0.22 ; \quad I^{2}=32 \% ; \quad Z=16.72\right.$, $p<0.00001$; -Fig. 6B).

\section{NT-proBNP and Survival}

There were two studies documenting plasma NT-proBNP levels as a continuous variable, which highlighted that the fatal cases had several times of upregulation in NT-proBNP expression compared with nonfatal ones $\left(\mathrm{Tau}^{2}=6.03 ; \mathrm{Chi}^{2}=2.41, \mathrm{df}=1\right.$, $p=0.12 ; I^{2}=59 \% ; Z=4.00 ; p<0.0001 ;$ - Fig. 7$)$.

\section{HFMD Control Mean Difference Mean Difference \\ Study or Subgroup Mean SD Total Mean SD Total Weight IV, Fixed, $95 \%$ C \\ IV, Fixed, $95 \% \mathrm{Cl}$

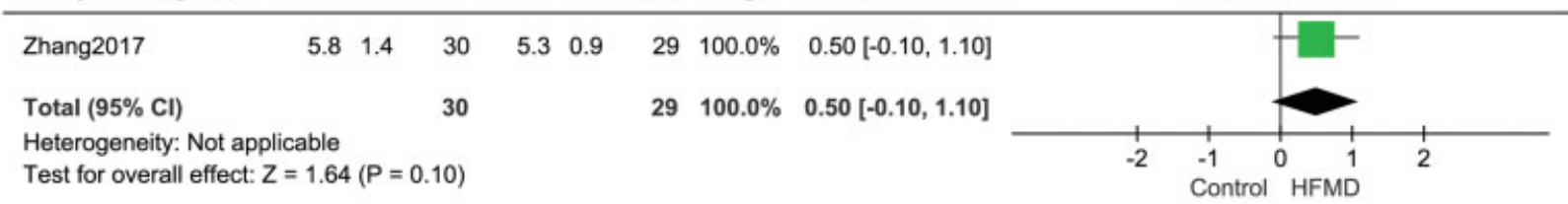

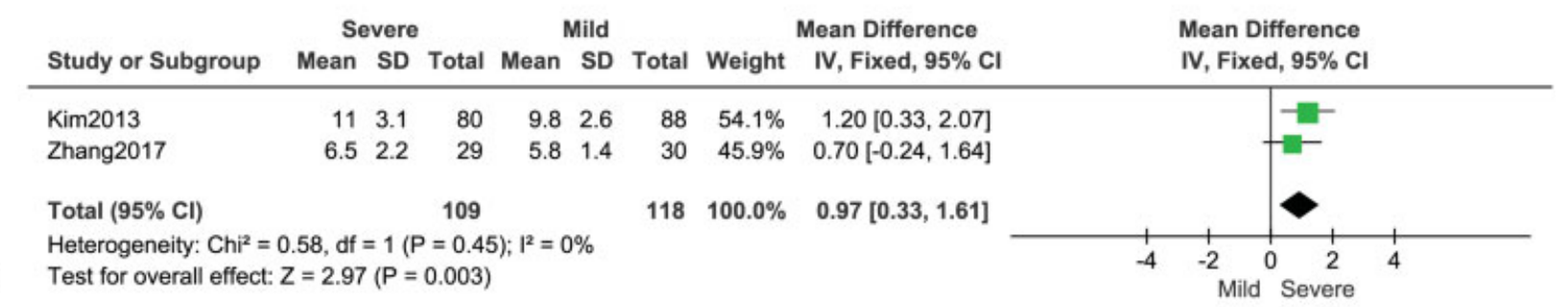

\begin{tabular}{|c|c|c|c|c|c|c|c|c|c|c|c|c|}
\hline \multirow[b]{2}{*}{ Study or Subgroup } & \multicolumn{3}{|c|}{ Dead } & \multicolumn{3}{|c|}{ Survived } & \multicolumn{3}{|c|}{ Mean Difference } & \multirow{2}{*}{\multicolumn{2}{|c|}{$\begin{array}{l}\text { Mean Difference } \\
\text { IV, Fixed, } 95 \% \mathrm{CI}\end{array}$}} & \\
\hline & Mean & SD & Total & Mean & SD & Total & Weight & IV, Fixed, $95 \% \mathrm{CI}$ & & & & \\
\hline Zheng2017 & 18.2 & 7.7 & 90 & 10.3 & 5.2 & 37 & $47.8 \%$ & $7.90[5.59,10.21]$ & & & - & \\
\hline Zhang2017 & 12.7 & 2.8 & 4 & 6.9 & 1.7 & 20 & $31.6 \%$ & $5.80[2.96,8.64]$ & & & & \\
\hline Lee2016 & 12.7 & 7.2 & 16 & 6.6 & 6.1 & 229 & $19.5 \%$ & $6.10[2.48,9.72]$ & & & & \\
\hline Huang2003 & 16.2 & 13.4 & 4 & 9.1 & 13.1 & 13 & $1.1 \%$ & $7.10[-7.84,22.04]$ & & & & \\
\hline Total $(95 \% \mathrm{CI})$ & & & 114 & & & 299 & $100.0 \%$ & $6.88[5.28,8.47]$ & & & & \\
\hline \multicolumn{9}{|c|}{$\begin{array}{l}\text { Heterogeneity: } \mathrm{Chi}^{2}=1.48, \mathrm{df}=3(\mathrm{P}=0.69) ; \mathrm{I}^{2}=0 \% \\
\text { Test for oyerall effect: } Z=8.44(\mathrm{P}<0.00001)\end{array}$} & $\frac{1}{-20}$ & -10 & 10 & 20 \\
\hline
\end{tabular}

Fig. 5 Cumulative meta-analysis of plasma glucose levels between different groups (presented as forest plots). (A) Difference in plasma glucose levels between HFMD and control groups. (B) Difference in plasma glucose levels between the severe and mild groups. (C) Difference in plasma glucose levels between fatal and nonfatal groups. HFMD, hand, foot and mouth disease. 


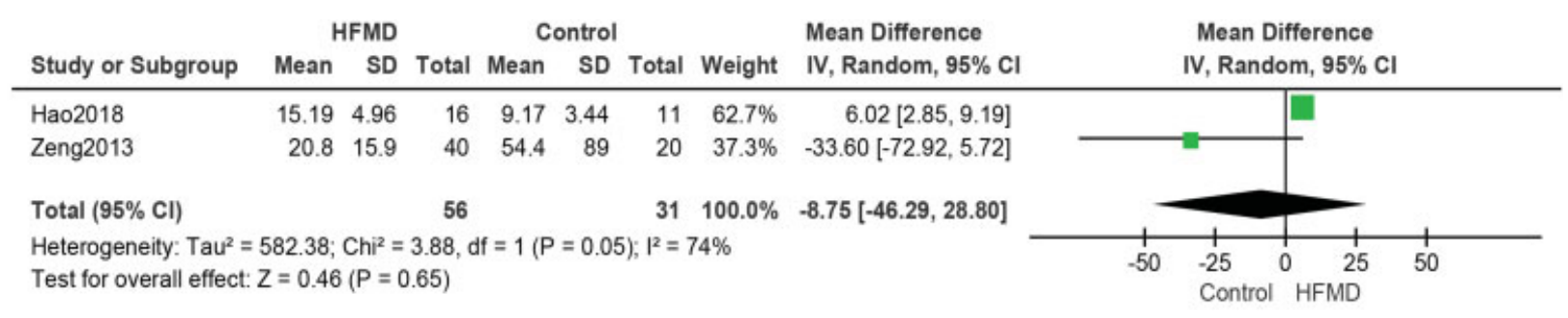

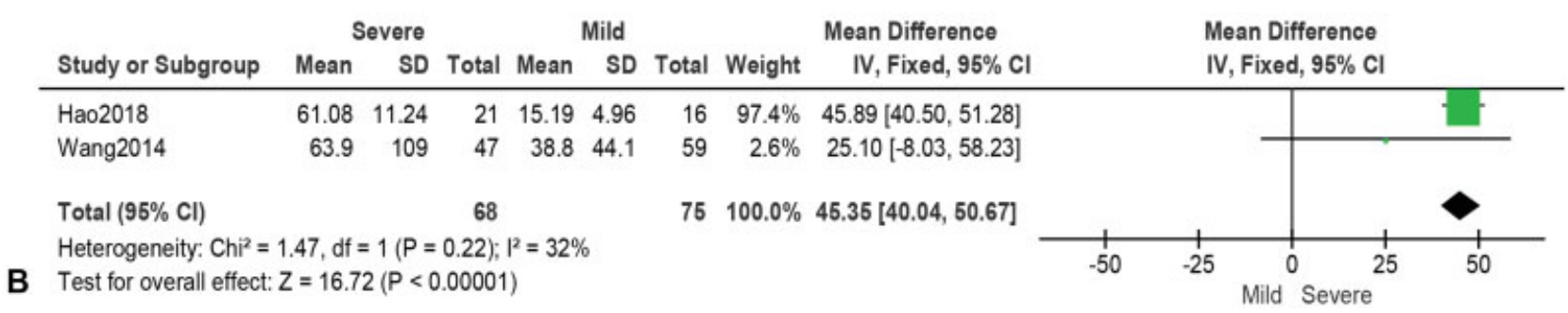

Fig. 6 Cumulative meta-analysis of IL-8 levels between different groups (presented as forest plots). (A) Difference in IL-8 levels between HFMD and control groups. (B) Difference in IL-8 levels between severe and mild groups. HFMD, hand, foot and mouth disease.

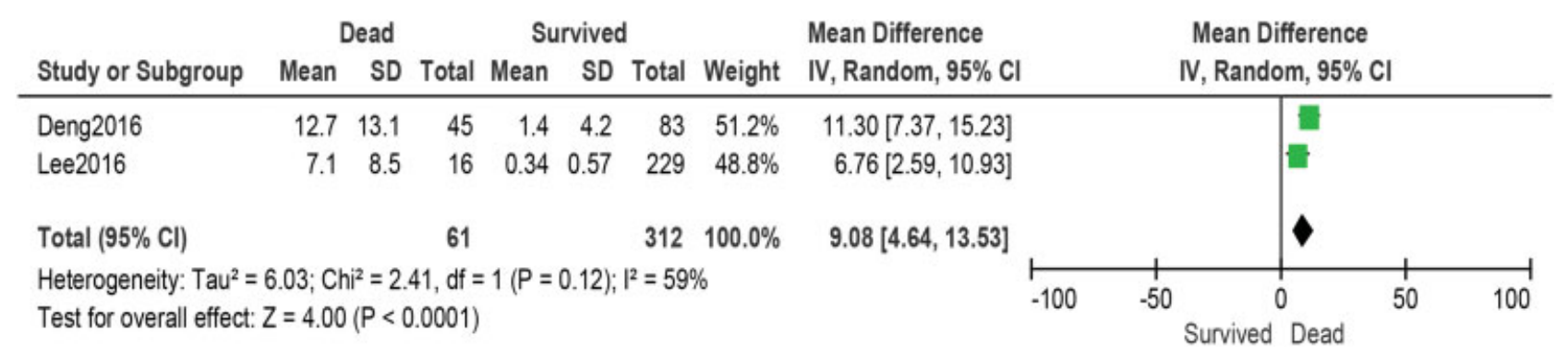

Fig. 7 Cumulative meta-analysis of plasma NT-proBNP levels between fatal and nonfatal groups. NT-proBNP, N-terminal pro-B-type natriuretic peptide.

Other Factors Associated with HFMD Grade and Ending Besides the above indicators that could feasibly be routinely applied, there were other blood markers with potential diagnostic/prognostic values. Lacking in the aggregation of the repeated studies, these studies could only be individually analyzed. Interferon $-\gamma($ IFN- $\gamma$ ) as one of the most widely studied immune-associated molecules, was significantly increased in HFMD patients compared with controls, ${ }^{31}$ and in the severe group compared with the mild group. ${ }^{22}$ Another cytokine IFN- $\alpha$ was lower in patients with complications than those without complications. Tumor necrosis factor- $\alpha$ (TNF- $\alpha$ ), one of the most widely studied inflammatory factors, was found to be highly increased in expression in HFMD cases. ${ }^{24}$ Also, serum amyloid A and clusterin were proposed to be potential predictive biomarkers for severe HFMD. ${ }^{18}$ Glutathione (GSH) and malondialdehyde (MDA) were another two useful indicators in HFMD evaluation. In the acute and recovery stages, HFMD patients showed a lower GSH level and higher MDA level compared with controls. ${ }^{20} \mathrm{D}$-dimmer is another coagulation indicator besides platelet. Zhang and Song observed that children with severe HFMD had increased plasma D-dimer levels, and the higher the levels, severer the condition. ${ }^{27}$ A recent study pointed that serum concentrations of angiotensin II and noradrenaline in HFMD patients were significantly higher than that in healthy controls. ${ }^{26}$ Other than carbohydrates and proteins, noncoding RNAs were also found impacted by HFMD development. Zhao et al pointed out that plasma circular DNAs can be an early identification marker of HFMD: their levels were increased in ordinary HFMD and even elevated in the severe group. ${ }^{29}$ Differential serum expression levels of ten miRNAs (miR-140-5p, miR-143, miR-148a, etc.) also changed in HFMD patients, by Cui et al. ${ }^{6}$ Lastly, some immune-related gene polymorphism (e.g., IL-10) was associated with the gene expression and severity of HFMD. ${ }^{28}$ Collectively, these reports (with detailed data of each parameter) have shown significance of some new but easily detectable markers, but they were majorly single ones in their focused indexes. More evidences are needed for better understanding of their diagnostic and prognostic values.

Finally, we found no publication bias for conclusions on funnel plot analysis, because significant symmetrical appearance was demonstrated.

\section{Discussion}

This meta-analysis used 26 studies and found some interesting factors with potential diagnostic and prognostic meanings in the peripheral blood. Specifically, WBC counts had an association with later survival; CRP and platelet levels had limited 
indicative roles; a higher plasma glucose level may imply graver complications and poorer survival; fatal outcome was correlated with increased IL-8 and NT-proBNP levels.

Ordinarily, EV71 is a leading common cause of HFMD, aseptic meningitis, and encephalitis in Asian populations. Application of reverse transcription polymerase chain reaction or sequencing technique to identify HEV71 amplicons was a direct method in HFMD diagnosis; at the protein level, EV71 and CVA1 neutralizing IgM antibodies have a high prognostic value for HFMD. So far, multiple assays have been proposed for detecting the EV71 antigen in HFMD serum samples; rapid and sensitive screening techniques have also been developed in recent years, such as rapid point-of-care tests ${ }^{32}$ and protein microarray-mediated detection techniques. ${ }^{33}$ However, these indicators are not among routine investigations, and only patients highly suspected of having HFMD could be recommended the above detections. Moreover, a limited sight of EV71/CVA1 markers cannot afford useful risk factors in routine blood tests, which may provide additional evaluation references without adding the diagnostic cost.

To date, large number of studies have observed potential risk factors in HFMD. Dominant publications focused on clinical manifestations, like vomiting, fever, and lethargy, etc. Also, Chinese scholars showed high interests in temperature and humidity. ${ }^{34-36}$ In the present study, we investigated the commonly used indexes in peripheral blood and suggested that these indexes can be potential assistant biomarkers in HFMD.

Intriguingly, $\mathrm{WBC}$ variations showed great heterogeneity among studies, and this factor has limited values in distinguishing HFMD/control or severe/mild. However, the WBC level around $18 \times 10^{9} / \mathrm{L}$ suggests a death outcome. Therefore, it is still worth paying attention to high WBC levels. In the present analysis, CRP and platelet did not show a clear indicative value, although some rare reports have implied these markers may be positively correlated with HFMD severities. For example, there was a case report of adult HFMD with a long-lasting elevated CRP level. ${ }^{37}$ In 2016, a survey reported that patients in the severe HFMD group had an increased high-sensitivity CRP level compared with the mild group. ${ }^{38}$ More data accumulation is required to establish definitively whether there are links between CRP and platelet levels and HFMD diagnosis and prognosis. The relationship between NT-proBNP and survival is supported by other studies (not included on our meta-analysis because there were insufficient data). For example, Qiu et al reported that patients with NT-proBNP $\geq 1,300 \mathrm{pg} / \mathrm{mL}$ had higher risk of death than those with NT-proBNp $<1,300 \mathrm{pg} / \mathrm{mL}^{39}$ This conclusion can be supported by our meta-analysis, and our finding strongly implied that several fold higher NT-proBNP is a sign of poor outcome.

The inflammatory cytokine IL- 8 was found dramatically elevated in the severe group in comparison with mild group based on this analysis, which suggests the progression of HFMD, as well as HFMD caused death, accomplished by above related indicators (WBC, glucose, and NT-proBNP), may be due to uncontrolled inflammatory responses. Some of our included studies did not provide the detailed ex- pression levels of cytokines, but they showed consistent results. Li et al observed that the levels of IL-1b, IL-6, IL-10, MIP-1, and TNF- $\alpha$ were higher in HFMD patients than controls, IL-6 and TNF- $\alpha$ levels were even higher in HFMD patients as compared with mild HFMD. ${ }^{17}$ A report in 2014 (not included in our analysis) also showed different cytokine profiles in different cohorts. In patients with mild HFMD, the peaks of IL-8 and IL-10 were observed on day 6 and that of IL-18 was observed on day 4; in those with severe illness, all cytokines spiked on day 3 and peaked on day 11; and cytokines were significantly correlated with immunoglobulin $\mathrm{M}$ levels by the end of the disease course. ${ }^{40}$ In another two studies, all kinds of cytokines/ chemokines were upregulated in severe HFMD patients compared with mild HFMD, but RANTES, MCP-1, IL-4, IL12 , and IL-18 levels were higher in mild HFMD patients than in the controls. ${ }^{41,42}$

There are some limitations in the present study. We have only identified 26 papers from four databases. Overall, the number of cases for many blood parameters was small. When the total references are limited, judgment may be biased due to expectations and misleading conclusions may be drawn. We hope increasing evidence to support our conclusion that routine blood markers may be useful will promote further studies.

In conclusion, some blood factors, especially WBC counts, plasma glucose level, IL-8, and NT-proBNP levels are associated with the severity and outcome of HFMD, but more evidences are needed to confirm these findings.

\section{Conflict of Interest}

None declared.

\section{References}

1 Koh WM, Bogich T, Siegel K, et al. The epidemiology of hand, foot and mouth disease in Asia: a systematic review and analysis. Pediatr Infect Dis J 2016;35(10):e285-e300

2 Kaminska K, Martinetti G, Lucchini R, Kaya G, Mainetti C. Coxsackievirus A6 and hand, foot and mouth disease: three case reports of familial child-to-immunocompetent adult transmission and a literature review. Case Rep Dermatol 2013;5(02): 203-209

3 Omaña-Cepeda C, Martínez-Valverde A, del Mar Sabater-Recolons M, Jané-Salas E, Marí-Roig A, López-López J. A literature review and case report of hand, foot and mouth disease in an immunocompetent adult. BMC Res Notes 2016;9:165

4 Chang LY, Hsia SH, Wu CT, et al. Outcome of enterovirus 71 infections with or without stage-based management: 1998 to 2002. Pediatr Infect Dis J 2004;23(04):327-332

5 Chen S, Yang Y, Yan X, Chen J, Yu H, Wang W. Influence of vitamin A status on the antiviral immunity of children with hand, foot and mouth disease. Clin Nutr 2012;31(04):543-548

6 Cui L, Qi Y, Li H, et al. Serum microRNA expression profile distinguishes enterovirus 71 and coxsackievirus 16 infections in patients with hand-foot-and-mouth disease. PLoS One 2011; 6(11):e27071

7 Deng HL, Zhang YF, Li YP, et al. N-terminal pro-brain natriuretic peptide levels associated with severe hand, foot and mouth disease. BMC Infect Dis 2016;16(01):585

8 Duan G, Yang H, Shi L, et al. Serum inflammatory cytokine levels correlate with hand-foot-mouth disease severity: a nested serial case-control study. PLoS One 2014;9(11):e112676 
9 Hao J, Li P, Tian Y, et al. Crosstalk between toll-like receptor 3 and notch signaling contributes to $\mathrm{CD} 14^{+}$monocytes activity in enterovirus 71 infected hand, foot, and mouth disease. Int Immunopharmacol 2018;60:26-33

10 Huang M, Du W, Liu J, et al. Interleukin-27 as a novel biomarker for early cardiopulmonary failure in enterovirus 71-infected children with central nervous system involvement. Mediators Inflamm 2016;2016:4025167

11 Huang YF, Chiu PC, Chen CC, et al. Cardiac troponin I: a reliable marker and early myocardial involvement with meningoencephalitis after fatal enterovirus-71 infection. J Infect 2003;46(04): 238-243

12 Kim SJ, Kim JH, Kang JH, et al; Enteroviruses Complications Working Group. Risk factors for neurologic complications of hand, foot and mouth disease in the Republic of Korea, 2009. J Korean Med Sci 2013;28(01):120-127

13 Lee CC, Lu X, Xiao Z, Yang M, Zhu Y. Prognostic value of B-type natriuretic peptide, leukocytosis, and hyperglycemia in children with severe hand, foot, and mouth disease. Shock 2016;45(06): 620-625

14 Li CC, Yang MY, Chen RF, et al. Clinical manifestations and laboratory assessment in an enterovirus 71 outbreak in southern Taiwan. Scand J Infect Dis 2002;34(02):104-109

15 Li S, Cai C, Feng J, et al. Peripheral T lymphocyte subset imbalances in children with enterovirus 71-induced hand, foot and mouth disease. Virus Res 2014;180:84-91

16 Li W, Teng G, Tong H, et al. Study on risk factors for severe hand, foot and mouth disease in China. PLoS One 2014;9(01):e87603

$17 \mathrm{Li} \mathrm{X}, \mathrm{Li} \mathrm{Q}, \mathrm{Li}$ J, et al. Elevated levels of circulating histones indicate disease activity in patients with hand, foot, and mouth disease (HFMD). Scand J Infect Dis 2014;46(12):818-824

18 Liu J, Huang P, He Y, et al. Serum amyloid A and clusterin as potential predictive biomarkers for severe hand, foot and mouth disease by 2D-DIGE proteomics analysis. PLoS One 2014;9(09): e108816

19 Liu JT, Peng D, Guan XH, Zou DD, Zhao DC. Clinical characteristics and treatment assessments of severe enterovirus 71 infected children [in Chinese]. Zhonghua Er Ke Za Zhi 2010;48(01):24-28

20 Ou JB, Zhang CM, Fu SM, Huang X, Huang LH. Relationship between G6PD deficiency and hand-foot-mouth disease induced by enterovirus 71 [in Chinese]. Zhongguo Dang Dai Er Ke Za Zhi 2013;15(09):751-755

21 Van Pham H, Hoang TNA, Duong HT, et al. Clinical characteristics of hand, foot and mouth disease in Daklak Province, Vietnam and associated factors of severe cases. Virusdisease 2017;28(04): 430-433

22 Wang W, Li W, Yang X, et al. Interleukin-8 is elevated in severe hand, foot, and mouth disease. J Infect Dev Ctries 2014;8(01): 94-100

$23 \mathrm{Wu}$ J, Cui D, Yang X, et al. Increased frequency of circulating follicular helper $\mathrm{T}$ cells in children with hand, foot, and mouth disease caused by enterovirus 71 infection. J Immunol Res 2014; 2014:651872

24 Zeng $\mathrm{M}$, Zheng $\mathrm{X}$, Wei R, et al. The cytokine and chemokine profiles in patients with hand, foot and mouth disease of different severities in Shanghai, China, 2010. PLoS Negl Trop Dis 2013;7 (12):e2599

25 Zhang A, Yang L, Guo P, et al. B-type brain natriuretic peptide as a measure of the severity of hand-foot-mouth disease: a casecontrol study. BMC Infect Dis 2017;17(01):651
26 Zhang C, Chen S, Zhou G, et al. Involvement of the renin-angiotensin system in the progression of severe hand-foot-and-mouth disease. PLoS One 2018;13(05):e0197861

27 Zhang SL, Song CF. Dynamic changes in plasma D-dimer level and its prognostic value in children with severe hand-foot- mouth disease [in Chinese]. Zhongguo Dang Dai Er Ke Za Zhi 2013;15 (12):1119-1122

28 Zhao N, Chen HL, Chen ZZ, Li J, Chen ZB. IL-10-592 polymorphism is associated with IL-10 expression and severity of enterovirus 71 infection in Chinese children. J Clin Virol 2017;95:42-46

29 Zhao ZX, Lu JH, Mei ZQ et al. The level of peripheral circular DNA in patients with hand foot and mouth disease [in Chinese]. Zhonghua Shi Yan He Lin Chuang Bing Du Xue Za Zhi 2011;25(06):483-485

30 Zheng G, Cao J, Yu J, Zhang Z, Liu Q, Chen J. Risk factors for death in children with critical and severe hand-foot-and-mouth disease in Chongqing, China: an observational study. Medicine (Baltimore) 2017;96(49):e8934

31 Tan Y, Yang T, Liu P, et al. Association of the OAS3 rs1859330 G/A genetic polymorphism with severity of enterovirus-71 infection in Chinese Han children. Arch Virol 2017;162(08):2305-2313

32 Zhang J, Weng Z, Du H, et al. Development and evaluation of rapid point-of-care tests for detection of Enterovirus 71 and Coxsackievirus A16 specific immunoglobulin M antibodies. J Virol Methods 2016;231:44-47

33 Zhang A, Xiu B, Zhang H, Li N. Protein microarray-mediated detection of antienterovirus antibodies in serum. J Int Med Res 2016;44(02):287-296

34 Coates SJ, Davis MDP, Andersen LK. Temperature and humidity affect the incidence of hand, foot, and mouth disease: a systematic review of the literature-a report from the International Society of Dermatology Climate Change Committee. Int J Dermatol 2019;58 (04):388-399

35 Chen SM, Du JW, Jin YM, et al. Risk factors for severe hand-footmouth disease in children in Hainan, China, 2011-2012. Asia Pac Public Health 2015;27(07):715-722

36 Long L, Gao LD, Hu SX, et al. Risk factors for death in children with severe hand, foot, and mouth disease in Hunan, China. Infect Dis (Lond) 2016;48(10):744-748

37 Toya M, Endo Y, Tanizaki H, Fujisawa A, Tanioka M, Miyachi Y. An adult case of severe hand-foot-mouth disease accompanying persistent fever and systemic arthritis. Dermatol Online J 2012; 18(08):14

38 Han FX, Gao JH, Gai JH. Clinical significance of combined liver function and high-sensitivity C-reactive protein measurement in children with hand-foot-mouth disease. Genet Mol Res 2016;15 (03)

39 Qiu J, Lu X, Liu X, et al. Derivation and validation of a mortality risk score for severe hand, foot and mouth disease in China. Sci Rep 2017;7(01):3371

40 Han J, Wang Y, Gan X, Song J, Sun P, Dong XP. Serum cytokine profiles of children with human enterovirus 71-associated hand, foot, and mouth disease. J Med Virol 2014;86(08): 1377-1385

41 Shang W, Qian S, Fang L, Han Y, Zheng C. Association study of inflammatory cytokine and chemokine expression in hand foot and mouth disease. Oncotarget 2017;8(45):79425-79432

42 Shao P, Wu X, Li H, Wu Z, Yang Z, Yao H. Clinical significance of inflammatory cytokine and chemokine expression in hand, foot and mouth disease. Mol Med Rep 2017;15(05): $2859-2866$ 